\title{
Pacific
}

Journal of

Mathematics

\section{BESOV SPACES, MEAN OSCILLATION, AND GENERALIZED HANKEL OPERATORS}

\author{
MARCo MARIA PELOSO
}




\title{
BESOV SPACES, MEAN OSCILLATION, AND GENERALIZED HANKEL OPERATORS
}

\section{Marco M. Peloso}

\begin{abstract}
We introduce some operators on the Bergman space $A^{2}$ on the unit ball that generalize the classical (big) Hankel operator. For such operators we prove boundedness, compactness, and Schattenideal property criteria. These extend known results. These new operators are defined in terms of a symbol. We prove in particular that for $2 \leq p<\infty$, these operators belong to the Schatten ideal $S_{p}$ if and only if the symbol $f$ is in the Besov space $B_{p}$. We also give several different characterizations of the norm on the Besov spaces $B_{p}$. In particular we prove that the Besov spaces are the mean oscillation spaces in the Bergman metric, for $1 \leq p<\infty$.
\end{abstract}

Consider the unit ball $B$ in $C^{n}$ and the Bergman space $A^{2}(B)$ of the holomorphic functions that are square integrable with respect to the (normalized) Lebesgue measure $d m$. The space $A^{2}$ admits a reproducing kernel $\mathscr{K}(z, w)$, the well-known Bergman kernel. Let $\mathscr{P}$ denote the orthogonal projection of $L^{2}(d m)$ onto $A^{2}$. The Hankel operator on $A^{2}$ with symbol $f, H_{f}$, is defined by

$$
\begin{aligned}
H_{f} g(z) & =(I-\mathscr{P})(\bar{f} g)(z) \\
& =\int_{B} \overline{(f(z)-f(w))} \mathscr{K}(z, w) g(w) d m(w) .
\end{aligned}
$$

In recent times, the Hankel operator $H_{f}$ first appeared in the context of Hardy spaces on the unit circle. It has been extensively studied by now, even on the Bergman spaces on the disc and on the ball. Important papers in this context are [1], [3] for the case $n=1$, and [2], [7], [19], and [21] and [23] for the case $n>1$. It is known that $H_{f}$ is bounded if and only if $f$ is in the Bloch space (see $\S 2$ ) and it is compact if and only if $f$ is in the little Bloch space. These results are due to Axler for the case of the unit disc, and to Arazy, Fisher, Janson, and Peetre, and independently to $\mathrm{Zhu}$, for the case of the ball. The Schatten ideal properties of $H_{f}$ have been studied too. Arazy, Fisher, and Peetre for $n=1$, [3], and independently four (groups of ) authors ([2], [7], [19] and [21]) have proved that for $c(n)<p<\infty$, the Hankel operator $H_{f}$ is in the Schatten von Neumann class $S_{p}$ 
(see 2.5) if and only if $f$ is in the Besov space $B_{p}$ (see (4)). Here $c(n)$ is a value depending on the (complex) dimension $n$ of the unit ball and it has been called "cut-off" in the literature. The cut-off $c(n)$ equals 1 for $n=1$ and $2 n$ for $n>1$. If $0<p \leq c(n)$ and $H_{f}$ is in the Schatten class $S_{p}$, then $H_{f}=0$ and $f$ is constant.

An essential tool in proving the above-mentioned results is a characterization of the norm in the Besov spaces. Namely for $p>c(n)$ (in particular, for $n>1$ if $p>2 n$ ) we have that the following are equivalent:

(i) $f \in B_{p}$;

(ii) $\int_{B} \int_{B}|f(z)-f(w)|^{p}|\mathscr{K}(z, w)|^{2} d m(z) d m(w)<\infty$.

Moreover, the Besov spaces can be described in geometrical terms, that is, in terms of oscillations over balls in the Bergman metric. Let $\rho>0$ be fixed, and for $\zeta \in B$, let $E_{\zeta}$ be the ball in the Bergman metric with center $\zeta$ and radius $\rho$. For $g$ locally integrable, define the mean of $g$ over $E_{\zeta}$ as

$$
g_{E_{\zeta}}=\frac{1}{\left|E_{\zeta}\right|} \int_{E_{\zeta}} g d m
$$

Here $\left|E_{\zeta}\right|$ denote the normalized Lebesgue measure of the ball $E_{\zeta}$. Define the mean oscillation of $g$ at $\zeta$ to be

$$
\mathrm{MO}_{\rho} g(\zeta)=\frac{1}{\left|E_{\zeta}\right|} \int_{E_{\zeta}}\left|g-g_{E_{\zeta}}\right| d m
$$

Then the following are equivalent for $p>2 n$ (see [22] for the case $n=1)$

(i) $f \in B_{p}$;

(ii) $\mathrm{MO}_{\rho} f \in L^{p}(d \lambda)$.

It is also easy to see that if (ii) above holds and $0<p \leq 2 n$ then $f$ has to be constant.

In this paper we investigate these phenomena, trying to explain what "happens" below the cut-off. We replace the expression $f(z)-f(w)$ by $f(w)-\sum_{|\alpha|<j} \partial^{\alpha} f(z) /(\alpha !)(w-z)^{\alpha}$ in all the different situations in which the former appears. The latter expression has a higher order of zero at $w=z$, that is, along the diagonal of $B \times B$. We introduce in this way what we call generalized Hankel operators of order $j$ and with symbol $f$. We denote them by $H_{f ; j}$ and $H_{f: j}^{\prime}$, explicitly:

$$
H_{f ; j}^{\prime} g(z)=\int_{B} \overline{\left(f(w)-\sum_{|\alpha|<j} \frac{\partial^{\alpha} f(z)}{\alpha !}(w-z)^{\alpha}\right)} \frac{g(w)}{(1-z \cdot \bar{w})^{n+1}} d m(w)
$$


and

$$
H_{f ; j} g(z)=\int_{B}-\left(f(z)-\sum_{|\alpha|<j} \frac{\partial^{\alpha} f(w)}{\alpha !}(z-w)^{\alpha}\right) \frac{g(w)}{(1-z \cdot \bar{w})^{n+1}} d m(w)
$$

These operators coincide with the classical one when the order $j$ is 1 . For these operators we prove boundedness and compactness criteria. In these cases the results are the same as in the classical case: the generalized Hankel operators are bounded if and only if the symbol is in the Bloch space, and they are compact if and only if the symbol is in the little Bloch space. For the range $2 \leq p<\infty$, we prove that these new operators belong to $S_{p}$ if and only if $b \in B_{p}$ when $p$ is in the range $2 n / j<p<\infty$. That is, we are able to lower the cut-off $2 n$ by increasing the order of zero on the diagonal in the expression given by the symbol. In this process, we characterize the norm on the Besov spaces in terms of double integrals against the modulus squared of the Bergman kernel, extending the description mentioned earlier. As a consequence we also prove that, for $1 \leq p<\infty$, the Besov spaces are the mean oscillation spaces in the Bergman metric.

The phenomenon of the cut-off for Hankel-type operators was first observed by Janson and Wolff in [10] in the context of commutators of singular integral operators. Later, Rochberg raised the question of finding Hankel operators with cut-off $1 / j, j$ a positive number. Recently, Janson, Peng, Rochberg, and Wu have studied some operators on the Bergman space on the unit disc, called intermediate or middle Hankel operators, with cut-off $1 / j$, where $j$ is the "order" of the operator. Their approach is different, and we refer the reader to the papers [9] and [13], and references therein.

The paper is organized as follows. In $\S 1$ we state the main results. Section 2 summarizes the known facts and definitions needed in the sequel. Section 3 contains the results about what we call higher moment conditions. This characterization of the Besov spaces is of its own interest, but also allows us to prove the theorem concerning the generalized Hankel operators. This is the content of $\S 4$. Section 5 contains the proof of one important estimate. Finally in $\S 6$ we prove that the Besov spaces can be characterized as mean oscillation spaces; we make few remarks and present some open questions.

Acknowledgments. It is a pleasure to express my gratitude to my thesis advisor Professor Steve Krantz. The main results of this paper were obtained during the preparation of my thesis, written at Washington University in St. Louis under his direction. I would like to thank 
him for his encouragement and help during and after the completion of my graduate studies.

1. Statement of the main results. Throughout this paper we denote by $B$ the unit ball in $\mathbf{C}^{n}$. For $z, w \in \mathbf{C}^{n}$ we write the inner product as

$$
z \cdot \bar{w}=\sum_{i=1}^{n} z_{i} \bar{w}_{i}
$$

The space of holomorphic functions on $B$ will be denoted by $\mathscr{H}(B)$ and the group of automorphisms of $B$ by Aut $B$. The group Aut $B$. consists of all biholomorphic self-maps of $B$ onto itself. The group Aut $B$ can be described as follows (see [14]). For any $\zeta \in B$ define $\varphi_{\zeta}$ by

$$
\varphi_{\zeta}=\frac{\zeta-P_{\zeta} z-\left(1-|z|^{2}\right)^{1 / 2} Q_{\zeta} z}{1-z \cdot \bar{\zeta}},
$$

where $P_{\zeta}$ is the orthogonal projection onto the subspace generated by $\zeta$ and $Q_{\zeta}=I-P_{\zeta}$. Then $\varphi_{\zeta} \in$ Aut $B$ and $\varphi_{\zeta}$ is an involution that interchanges $\zeta$ with the origin. Moreover,

$$
\text { Aut } B=\left\{\varphi_{\zeta} \circ U: \zeta \in B, U \in \mathscr{U}\right\} \text {, }
$$

where $\mathscr{U}$ is the space of unitary transformations of $\mathbf{C}^{n}$.

The normalized Lebesgue measure on $B$ will be denoted by $d m$, and the invariant volume form by $d \lambda$. Explicitly,

$$
d \lambda(z)=\frac{d m(z)}{\left(1-|z|^{2}\right)^{n+1}}
$$

(see [14]).

The gradient of a holomorphic function $f$ will be denoted by $\partial f$, that is,

$$
\partial f=\left(\partial_{1} f, \ldots, \partial_{n} f\right)
$$

where

$$
\partial_{j} f=\frac{\partial f}{\partial z_{j}} .
$$

Moreover we will use the following notations. We write

$$
\partial^{\alpha} f(z)=\frac{\partial^{\alpha} f}{\partial z^{\alpha}}(z)
$$

where $\alpha$ is a multi-index. The radial derivative of $f \in \mathscr{H}(B)$ is defined to be

$$
R f(z)=z \cdot \partial f(z)=\sum_{i=1}^{n} z_{i} \partial_{i} f(z)
$$


The Bergman space $A^{2}$ is the closed subspace of $L^{2}(\mathrm{dm})$ that consists of the holomorphic functions. We write $\mathscr{K}(z, w)$ to denote the Bergman kernel, that is, the reproducing kernel of $A^{2}(B)$, and $\mathscr{P}$ to denote the Bergman projection. Explicitly, for any $g \in L^{2}(d m)$,

$$
\mathscr{P} g(z)=\int_{B} g(w) \frac{1}{(1-z \cdot \bar{w})^{n+1}} d m(w) \text {. }
$$

The (diagonal) Besov spaces of analytic functions $B_{p}, 0<p<\infty$, are defined by

$$
B_{p}=\left\{f \in \mathscr{H}(B):\left(1-|z|^{2}\right)^{m} R^{m} f(z) \in L^{p}(d \lambda)\right\},
$$

where $m>n / p$. We remark that the Besov spaces $B_{p}$ defined here are the spaces $B_{p}^{n / p}$ (or $B_{p}^{n / p, 1 / p}$ ) of the scale of weighted Besov spaces (see [11] or [12]). We will not pursue the weighted case in this paper, and we consider only the spaces defined in (4).

Definition 1.1. For any $f \in \mathscr{H}(B), j$ a positive integer, and for $z, w \in B$, we set

$$
\Delta_{j} f(w, z)=f(w)-\sum_{|\alpha|<j} \frac{\partial^{\alpha} f(z)}{\alpha !}(w-z)^{\alpha} .
$$

Notice the different roles played by the variables $w$ and $z$. The first should be interpreted as the variable, and the second as the base point for the Taylor expansion.

We are ready to state our first main result.

THEOREM 1.2. Let $0<p<\infty, n \geq 2$. Let $j$ be a positive integer. If $j>2 n / p$ the following are equivalent.

(i) $\|f\|_{B_{p}}<\infty$.

(ii) $\int_{B} \int_{B} \frac{\left|\Delta_{j} f(w, z)\right|^{p}}{(1-z \cdot \bar{w})^{2(n+1)}} d m(z) d m(w)<\infty$.

If $j \leq 2 n / p$ and $f \in \mathscr{H}(B)$ satisfies (ii), then $f$ is a polynomial of degree at most $j-1$.

REMARK 1.3. A version of the above theorem holds for $n=1$. In this case the integer $j$ must satisfy the inequality $j>1 / p$. This phenomenon will carry over through the whole paper. The general reading key is that one should replace " $2 n$ " with " 1 " when $n=1$. This is a well-known fact by now. It depends essentially on the shape of the balls in the Bergman metric.

Even if not stated explicitly, all the results proved for $n>1$ carry over to the case $n=1$, with the convention mentioned above. 
REMARK 1.4. The above theorem is an important result. It gives an equivalent norm for all Besov spaces in terms of a double integral against the modulus squared of the Bergman kernel. It gives a natural extension to the case $j>1$ and $0<p \leq 2 n$ of the results in [2], [22], [19], and [21] where they treat the case $j=1$.

We now introduce the generalized Hankel operators on the Bergman space $A^{2}(B)$.

Definition 1.5. Let $j$ be a positive integer. Let $f \in \mathscr{H}(B)$ and such that $\partial^{\alpha} f \in L^{1}(d m)$ for $|\alpha|<j$. For $g \in L^{\infty} \cap \mathscr{H}(B)$, we define the generalized Hankel operators of order $j$ and with symbol $f, H_{f ; j}$ and $H_{f ; j}^{\prime}$ by

$$
H_{f ; j} g(z)=\int_{B} \overline{-\Delta_{j} f(w, z)} \mathscr{K}(z, w) g(w) d m(w),
$$

and

$$
H_{f ; j}^{\prime} g(z)=\int_{B} \overline{\Delta_{j} f(z, w)} \mathscr{K}(z, w) g(w) d m(w) \text {. }
$$

If $H_{f ; j}$ and $H_{f ; j}^{\prime}$ turn out to be bounded on $A^{2}$, we can extend the domain of the operators to all of $A^{2}$.

REMARK 1.6. Notice that if $j=1, H_{f ; 1}=H_{f ; 1}^{\prime}=H_{f}$, where $H_{f}$ is the classical Hankel operator on $A^{2}(B)$.

For the generalized Hankel operators our main theorems are the following.

Theorem 1.7. Let $f \in \mathscr{H}(B), j$ be any positive integer. Then the following are equivalent.

(i) $H_{f ; j}$ is bounded.

(ii) $H_{f ; j}^{\prime}$ is bounded.

(iii) $f \in \mathscr{B}$.

Theorem 1.8. Let $f \in \mathscr{H}(B), j$ be any positive integer. Then the following are equivalent.

(i) $H_{f ; j}$ is compact.

(ii) $H_{f ; j}^{\prime}$ is compact.

(iii) $f \in \mathscr{B}_{0}$.

Theorem 1.9. Let $n \geq 2, f \in \mathscr{H}(B)$, and let $2 \leq p<\infty$. Let $j$ be any positive integer. If $j>2 n / p$, then the following are equivalent.

(i) $H_{f ; j} \in S_{p}$. 
(ii) $H_{f ; j}^{\prime} \in S_{p}$.

(iii) $f \in B_{p}$.

If $j \leq 2 n / p$ and $H_{f ; j}$ or $H_{f ; j}^{\prime} \in S_{p}$, then $H_{f ; j}=H_{f ; j}^{\prime}=0$ and $f$ is a polynomial of degree $j-1$ at most.

REMARK 1.10. The above theorem is an extension of the results about $S_{p}$-properties of the classical Hankel operators on the Bergman spaces, as proved in the already mentioned papers [3], [2], [7], [19], and [21]. Theorem 1.9 describes the phenomenon of the cut-off in some detail. We should interpret the order $j$ as the order of zero along the diagonal of the term $\Delta_{j} f$ defined by the symbol $f$. As $j$ grows we obtain better $S_{p}$-properties for the operators $H_{f ; j}$ and $H_{f ; j}^{\prime}$.

We conclude this section by stating our theorems about the mean oscillation spaces in the Bergman metric.

Let $\rho>0$, and let $E_{\zeta}=E(\zeta, \rho)$ be the ball in the Bergman metric with center $\zeta$ and radius $\rho$. Recall that in (1) we have defined the mean of a locally integrable function over $E_{\zeta}$.

Definition 1.11. Let $f \in \mathscr{H}(B)$ and let $j$ be a positive integer. We define the mean oscillation of order $j$ of $f$ at $\zeta$ to be

$$
\mathrm{MO}_{\rho}^{j} f(\zeta)=\frac{1}{\left|E_{\zeta}\right|} \int_{E_{\zeta}}\left|\Delta_{j} f(w, \zeta)-\left(\Delta_{j} f(\cdot, \zeta)\right)_{E_{\zeta}}\right| d m(w) .
$$

THEOREM 1.12. Let $1 \leq p<\infty$, and let $j$ be a positive integer. Let $f \in \mathscr{H}(B)$ and $\mathrm{MO}_{\rho}^{j} f$ be defined as in 1.11. Then there exists $\rho_{0}>0$ such that for $0<\rho<\rho_{0}$ the following hold. For $j>2 n / p$ the following are equivalent.

(i) $f \in B_{p}$.

(ii) $\mathrm{MO}_{\rho}^{j} f \in L^{p}(d \lambda)$.

If $j \leq 2 n / p$ and $\mathrm{MO}_{\rho}^{j} f \in L^{p}(d \lambda)$, then $f$ is a polynomial of degree at most $j-1$.

Again this result extends the previous results by Zhu to the case (of the ball, and) to the case $1 \leq p \leq 2 n$ and $j>1$.

2. Preliminaries. For a $C^{1}$-function $g$ we denote its gradient by $\nabla g$. We define the invariant derivative of $g$ by setting

$$
\widetilde{D} g(z)=\left|\nabla\left(g \circ \varphi_{z}\right)(0)\right| \text {. }
$$


Definition 2.1. For $i, j=1, \ldots, n$ define the differential operators $T_{i j}$ by

$$
T_{i j}=\bar{z}_{i} \partial_{j}-\bar{z}_{j} \partial_{i}
$$

Then $T_{i j}$ is tangential, in the sense that $T_{i j}\left(|z|^{2}\right)=0$. For $C^{1}$ function $g$ we define the tangential derivative $D_{\tau} g$ by

$$
D_{\tau} g(z)^{2}=\sum_{i j}\left|T_{i j} g(z)\right|^{2} .
$$

When $k \geq 2$ and $g \in C^{k}$ we set

$$
D_{\tau}^{k} g(z)^{2}=\sum_{i_{1}, j_{1}, \ldots, i_{k}, j_{k}}\left|T_{i_{1} j_{1}} \cdots T_{i_{k} j_{k}} g(z)\right|^{2} .
$$

Then $D_{\tau}$ measures the size of $\nabla g$ in the complex tangential directions. For $f \in \mathscr{H}(B)$ we have the following identities (recall that we write $\partial f$ for $\nabla f$ if $f$ is holomorphic).

$$
\begin{aligned}
|z|^{2}|\partial f(z)|^{2} & =\left|\sum_{i=1}^{n} z_{i} \partial_{i} f(z)\right|^{2}+\sum_{i, j=1}^{n}\left(|z|^{2} \delta_{i j}-z_{i} \bar{z}_{j}\right) \partial_{i} f(z) \overline{\partial_{j} f(z)} \\
& =|R f(z)|^{2}+D_{\tau} f(z)^{2} .
\end{aligned}
$$

We can relate the radial and tangential derivative to the invariant derivative. Using Möbius invariance it is easy to see that for $f \in$ $\mathscr{H}(B)$

$$
\begin{aligned}
\widetilde{D} f(z)^{2} & =\left|\partial\left(f \circ \varphi_{z}\right)(0)\right|^{2} \\
& =\left(1-|z|^{2}\right)\left(|\partial f(z)|^{2}-|R f(z)|^{2}\right) \\
& =\frac{1}{|z|^{2}}\left(\left(1-|z|^{2}\right)^{2}|R f(z)|^{2}+\left(1-|z|^{2}\right) D_{\tau} f(z)^{2}\right) .
\end{aligned}
$$

An application of the classical Hardy's lemma (see [8], Theorem 330, or [2], Lemma 3.3) gives the next result. Set

$$
\left|\partial^{k} f(z)\right|^{2} \equiv \sum_{|\alpha|=k}\left|\partial^{\alpha} f(z)\right|^{2}
$$

LEMMA 2.2. Let $f \in \mathscr{H}(B)$ be such that $\partial^{\alpha} f(0)=0$ for $|\alpha|<k$. Then for $0<p<\infty, s>-1$, there exists a constant $C>0$ such that

$$
\int_{B}\left(1-|z|^{2}\right)^{s}|f(z)| d m(z) \leq C \int_{B}\left(1-|z|^{2}\right)^{s+k p}\left|\partial^{k} f(z)\right|^{p} d m(z) .
$$

Proof. The case $k=1$ is just Lemma 3.3 together with Remark 3.2 in [2]. Now it suffices to iterate the same argument using the fact that $\partial^{\alpha} f(0)=0$ for $|\alpha|<k$. 
The Bloch space $\mathscr{B}$ on $B$ has been introduced by Timoney in [17] and [18], and then studied intensively by many authors. The space $\mathscr{B}$ is defined as the space of holomorphic functions $f$ for which $\widetilde{D} f$ is bounded in $B$. The little Bloch space $\mathscr{B}_{0}$ is defined to be the subspace of functions $f \in \mathscr{B}$ for which $\widetilde{D} f(z) \rightarrow 0$ as $|z| \rightarrow 1^{-}$.

Recall that in (4) we have defined the Besov spaces $B_{p}$ by

$$
B_{p}=\left\{f \in \mathscr{H}(B): \int_{B}\left(\left(1-|z|^{2}\right)^{m}\left|R^{m} f(z)\right|\right)^{p} d \lambda(z)<\infty\right\} .
$$

Here $m$ is any integer such that $m p>n$. It is well known that the definition of $B_{p}$ is independent of $m$, and that one can replace $\left|R^{m} f(z)\right|$ with $\left|\partial^{m} f(z)\right|$ in the definition of the Besov spaces (recall the notation (6)). We want to describe the atomic decomposition of the Besov spaces $B_{p}$, as obtained by Coifman and Rochberg in [6] (see [12] for some details).

TheOREM 2.3 (Coifman and Rochberg). Let $0<p<\infty$. Let

$$
\nu>\max \left(\frac{n}{p}(p-1), 0\right) .
$$

Then there exists a positive number $\vartheta_{0}=\vartheta_{0}(p, \nu, n)$ and a constant $C$ such that if the points $\left\{\zeta_{i}\right\}$ form a $\vartheta$-lattice with $\vartheta<\vartheta_{0}$ then the following hold.

(A) If $f \in B_{p}$ there exist numbers $\left\{c_{i}\right\}$ such that

$$
f(z)=\sum_{1}^{\infty} c_{i} \frac{\left(1-|\zeta|^{2}\right)^{\nu}}{\left(1-z \cdot \bar{\zeta}^{i}\right)^{\nu}}
$$

and

$$
\sum_{1}^{\infty}\left|c_{i}\right|^{p} \leq\|f\|_{B_{p}}^{p} .
$$

(B) If $\sum_{1}^{\infty}\left|c_{i}\right|^{p}<\infty$ then the function defined in (A) is in $B_{p}$ and

$$
\|f\|_{B_{p}}^{p} \leq \sum_{1}^{\infty}\left|c_{i}\right|^{p} .
$$

We recall the Forelli-Rudin estimate (see [14], 1.4.10).

Proposition 2.4. Let $s>-1, t \in \mathbf{R}$. Define

$$
I_{t}(z)=\int_{B} \frac{\left(1-|w|^{2}\right)^{s}}{|1-z \cdot \bar{w}|^{n+1+s+t}} d m(w) .
$$


Then, as $|z| \rightarrow 1^{-}, I_{t}$ is bounded if $t<0, I_{t}$ is asymptotic to $\log \left(1-|z|^{2}\right)^{-1}$ if $t=0$, and asymptotic to $\left(1-|z|^{2}\right)^{-t}$ if $t>0$.

Finally, we define the Schatten ideal $S_{p}$.

Definition 2.5. Let $1<p<\infty$. Let $H$ be a Hilbert space and let $T$ be a compact operator on $H$. For any integer $k$ define the $s(k)$-number of $T$ by

$$
s(k)=\inf \{\|T-F\|: \operatorname{rank}(F) \leq k\} .
$$

Then we say that $T \in S_{p}$ if

$$
\sum_{k=1}^{\infty} s(k)^{p}<\infty .
$$

In this case we set

$$
\|T\|_{S_{p}}=\left(\sum_{k=1}^{\infty} s(k)^{p}\right)^{1 / p} .
$$

3. Higher moment conditions. Recall that in (1.1) we have defined the $j$ th difference of $f$ at $z$ by

$$
\Delta_{j} f(w, z)=f(w)-\sum_{|\alpha|<j} \frac{\partial^{\alpha} f(z)}{\alpha !}(w-z)^{\alpha} .
$$

Now let $d \Lambda(z, w)$ be the measure on $B \times B$ defined by

$$
d \Lambda(z, w)=\frac{1}{|1-z \cdot \bar{w}|^{2(n+1)}} d m(z) d m(w) .
$$

In the next proposition recall the note made in 1.3.

Proposition 3.1. Let $n>1,0<p<\infty, j$ be an integer, $j>$ $2 n / p$. Then there exists a constant $C=C(j, p, n)$ such that for all $f \in B_{p}$ we have

$$
\left\|\Delta_{j} f\right\|_{L^{p}(d \Lambda)} \leq C\|f\|_{B_{p}} .
$$

Although this result is essential in the sequel, the proof is rather long and (somewhat) technical. It is therefore postponed until $\S 5$. However, some of the techniques involved might be of interest of their own.

Recall that we have set

$$
\left|\partial_{w}^{k} g\right|^{2}=\sum_{|\alpha|=k}\left|\frac{\partial^{\alpha} g}{\partial w^{\alpha}}\right|^{2} .
$$


Proposition 3.2. Let $n>1,0<q<\infty$ and $j$ be any positive integer. Then for $f \in \mathscr{H}(B)$ we have that

$$
\left(1-|z|^{2}\right)^{j}\left|R^{j} f(z)\right| \leq C\left(\int_{B}\left|\Delta_{j} f\left(\varphi_{z}(w), z\right)\right|^{q} d(w)\right)^{1 / q},
$$

and

$$
\left(1-|z|^{2}\right)^{j}\left|R^{j} f(z)\right| \leq C\left(\int_{B}\left|\Delta_{j} f\left(z, \varphi_{z}(w)\right)\right|^{q} d m(w)\right)^{1 / q},
$$

for some constant $C$ independent of $f$.

Proof. Recall that $\Delta_{j} f\left(\varphi_{z}(w), z\right)$ vanishes of order $j$ at $\varphi_{z}(w)=$ $z$, i.e. at $w=0$. Therefore taking $j$ derivatives of $\Delta_{j} f\left(\varphi_{z}(\cdot), z\right)$ and evaluating at $w=0$, in order to obtain non-zero terms, we need to land all derivatives on $f$, not on the terms coming from the chain rule. Moreover, we use the identity

$$
\varphi_{z}^{\prime}(0)=-\left(1-|z|^{2}\right) P_{z}-\left(1-|z|^{2}\right)^{1 / 2} Q_{z},
$$

as in [14], 2.2.2. Thus,

$$
\left|\partial_{w}^{j} \Delta_{j} f\left(\varphi_{z}(w), z\right)\right|_{w=0}\left|\approx \sum_{i=0}^{j}\left(1-|z|^{2}\right)^{j-i / 2}\right| D_{\tau}^{i} R^{j-i} f(z) \mid .
$$

In an analogous manner, it is easy to see that

$$
\frac{\partial}{\partial w_{k}} \Delta_{j} f\left(z, \varphi_{z}(w)\right)=\frac{\partial \varphi_{z}}{\partial w_{k}}(w) \cdot \sum_{|\alpha|=j-1} \frac{\partial^{\alpha} \partial f}{\alpha !}\left(\varphi_{z}(w)\right)\left(z-\varphi_{z}(w)\right)^{\alpha} \text {. }
$$

Therefore, applying the same observation as above, we see that

$$
\left|\partial_{w}^{j} \Delta_{j} f\left(z, \varphi_{z}(w)\right)\right|_{w=0}\left|\approx \sum_{i=0}^{j}\left(1-|z|^{2}\right)^{j-i / 2}\right| D_{\tau}^{i} R^{j-i} f(z) \mid .
$$

Now, write $F(w, z)$ for any of the expressions $\Delta_{j} f(w, z)$ or $\Delta_{j} f(z, w)$. By the Cauchy estimates it follows that

$$
\begin{aligned}
\left(1-|z|^{2}\right)^{j}\left|R^{j} f(z)\right| & \leq\left|\partial_{w}^{j} F\left(\varphi_{z}(w), z\right)\right|_{w=0} \mid \\
& \leq C\left(\int_{B}\left|F\left(\varphi_{z}(w), z\right)\right|^{q} d m(w)\right)^{1 / q} .
\end{aligned}
$$


Theorem 3.3. Let $n>1$, and $0<p<\infty$. Let $0<q \leq p$ and $j$ be a positive integer satisfying $j>2 n / p$. Then the following are equivalent for $f \in \mathscr{H}(B)$.

(i) $\left\|\Delta_{j} f\left(\varphi_{z}(\cdot), z\right)\right\|_{L^{q}(d m)} \in L^{p}(d \lambda)$.

(ii) $\left\|\Delta_{j} f\left(z, \varphi_{z}(\cdot)\right)\right\|_{L^{q}(d m)} \in L^{p}(d \lambda)$.

(iii) $\int_{B} \int_{B}\left|\Delta_{j} f(w, z)\right|^{p}|\mathscr{K}(z, w)|^{2} d m(z) d m(w)<\infty$.

(iv) $f \in B_{p}$.

If $j \leq 2 n / p$ and $f$ satisfies one of the conditions (i)-(iii), then $f$ is a polynomial of degree at most $j-1$.

Proof. Again, write $F(w, z)$ for either expression $\Delta_{j} f(w, z)$ or $\Delta_{j} f(z, w)$. If $q \leq p$, by 3.2 and by Jensen inequality it follows that

$$
\begin{aligned}
\int_{B}\left(\left(1-|z|^{2}\right)^{j}\left|R^{j} f(z)\right|\right)^{p} d \lambda(z) & \leq c \int_{B}\left\|F\left(\varphi_{z}(\cdot), z\right)\right\|_{L^{q}(d m)}^{p} d \lambda(z) \\
& =c \int_{B} \int_{B}\left|\Delta_{j} f(\eta, z)\right|^{p} d \Lambda(z, \eta),
\end{aligned}
$$

where we have performed the change of variables $\eta=\varphi_{z}(w)$ in the inner integral. Recall that the Jacobian of the above transformation is

$$
\left(\frac{1-|z|^{2}}{|1-\eta \cdot \bar{z}|^{2}}\right)^{n+1}
$$

(see [14] 2.2.6). Thus, we have proved that

$$
\text { (iii) } \Rightarrow(\text { i) } \Rightarrow \text { (iv), }
$$

and

$$
\text { (iii) } \Rightarrow(\text { ii }) \Rightarrow(\text { iv) } \text {. }
$$

Now, 3.1 gives that (iv) $\Rightarrow$ (iii) .

We only need to prove the statement when $j \leq 2 n / p$. It suffices to notice that if

$$
\left\|F\left(\varphi_{z}(\cdot), z\right)\right\|_{L^{q}(d m)} \in L^{p}(d \lambda)
$$

then $\Delta_{j} f \equiv 0$. By subharmonicity and 3.2 again we have that

$$
\begin{aligned}
\left\|F\left(\varphi_{z}(\cdot), z\right)\right\|_{L^{q}(d m)} & \geq\left|\partial_{w}^{j} F\left(\varphi_{z}(w), z\right)\right|_{w=0} \mid \\
& \geq\left(1-|z|^{2}\right)^{j / 2} D_{\tau}^{j} f(z) .
\end{aligned}
$$

Thus,

$$
\int_{B}\left(1-|z|^{2}\right)^{j p / 2} D_{\tau}^{j} f(z)^{p} d \lambda(z)
$$


must be finite. If we assume (without loss of generality) that $f$ is holomorphic across the boundary, it follows that

$$
D_{\tau}^{j} f \equiv 0 .
$$

Simple considerations now show that $f$ is a polynomial of degree less than $j$.

We want to prove a version of the previous theorem for the case $p=\infty$. We need few modifications.

THeOREM 3.4. Let $f \in \mathscr{H}(B), j$ be a positive integer, $0<q<\infty$. Then the following quantities are equivalent.

$$
\begin{aligned}
\mathrm{I} & :=\sup _{\zeta \in B}\left(1-|\zeta|^{2}\right)^{j}\left|R^{j} f(\zeta)\right| . \\
\mathrm{II} & :=\sup _{\zeta \in B}\left(\int_{B}\left|\Delta_{j} f\left(\varphi_{\zeta}(w), \zeta\right)\right|^{q} d m(w)\right)^{1 / q} .
\end{aligned}
$$

REMARK. The case $j=1$ of the theorem is proved in [1]; similar results are proved in [16].

Proof. By 3.2 it follows that

$$
\left(1-|\zeta|^{2}\right)^{j}\left|R^{j} f(\zeta)\right| \leq c\left(\int_{B}\left|\Delta_{j} f\left(\varphi_{\zeta}(w), \zeta\right)\right|^{q} d m(w)\right)^{1 / q}
$$

Thus,

$$
\mathrm{I} \lesssim \mathrm{II}
$$

Write $F$ for $\Delta_{j} f$. Since $F\left(\varphi_{\zeta}(w), \zeta\right)$ has a zero of order $j$ at $w=0$, we can apply Hardy's Lemma 2.2.

$$
\begin{aligned}
& \left\|F\left(\varphi_{\zeta}(\cdot), \zeta\right)\right\|_{L^{p}(d m)}^{p} \\
& \quad \leq \int_{B}\left(1-|w|^{2}\right)^{j p}\left|\partial_{w}^{j} F\left(\varphi_{\zeta}(w), \zeta\right)\right|^{p} d m(\zeta) \\
& \quad \leq c \sup _{\xi \in B} \widetilde{D}\left(F\left(\varphi_{\zeta}(\cdot), \zeta\right)\right)(\xi)=c \sup _{\xi \in B} \widetilde{D}(F(\cdot, \zeta))(\xi),
\end{aligned}
$$

where we have used the equality

$$
\sup _{\xi \in B} \widetilde{D}(g \circ \psi)=\sup _{\xi \in B} \widetilde{D} g .
$$


Now it follows that

$$
\|F(\cdot, \zeta)\|_{\mathscr{B}} \leq \sup _{z \in B}\left(1-|z|^{2}\right)\left|\partial_{z}(F(z, \zeta))\right| \leq c\|f\|_{\mathscr{B}} .
$$

This shows that

$$
\mathrm{II} \lesssim \mathrm{I}
$$

Hence, we are done.

COROllary 3.5. Let $f \in \mathscr{H}(B)$. Let $j$ be any positive integer, $0<p<\infty$, and $\rho>0$. Then the following are equivalent.

(i) $f \in \mathscr{B}_{0}$.

(ii) $\lim _{|\zeta| \rightarrow 1}\left\|\Delta_{j} f\left(\varphi_{\zeta}(\cdot), \zeta\right)\right\|_{L^{p}(d m)}=0$.

Proof. This is actually a corollary of the proof of 3.4. Recall the notation there introduced. We have shown that

$$
\mathrm{I} \lesssim \mathrm{II}
$$

Thus, (ii) $\Rightarrow$ (i). Assume that (i) holds, and let $f$ be holomorphic across the boundary. Then (ii) holds as a consequence of the Lebesgue dominated convergence theorem. Now a density argument finishes the proof.

4. Generalized Hankel operators. In this section we prove the main theorems about the generalized Hankel operators. Recall that we have defined the operators $H_{f ; j}$ and $H_{f ; j}^{\prime}$ on $A^{2}$ by setting

$$
H_{f ; j} g(z)=\int_{B} \overline{-\Delta_{j} f(w, z)} \mathscr{K}(z, w) g(w) d m(w),
$$

and

$$
H_{f ; j}^{\prime} g(z)=\int_{B} \overline{\Delta_{j} f(z, w)} \mathscr{K}(z, w) g(w) d m(w) .
$$

The operators $H_{f ; j}$ and $H_{f ; j}^{\prime}$ share many important properties with the classical big Hankel operators. Some of these are illustrated in the propositions that follow. Write $\left(A^{2}\right)^{\perp}$ to indicate the orthogonal complement of $A^{2}$ in $L^{2}(d m)$.

Lemma 4.1. Let $j$ be any positive integer. Then

$$
H_{f ; j}, H_{f ; j}^{\prime}: A^{2} \rightarrow\left(A^{2}\right)^{\perp} .
$$

Proof. Same as in the classical case. 
LEMMA 4.2. Write $\mathscr{K}_{\zeta}(z)=\mathscr{K}(z, \zeta)$. Then for any positive integer $j$

and

$$
H_{f ; j} \mathscr{K}_{\zeta}=\overline{-\Delta_{j} f(\zeta, \cdot)} \mathscr{K}_{\zeta},
$$

$$
H_{f ; j}^{\prime} \mathscr{K}_{\zeta}=\overline{\Delta_{j} f(\cdot, \zeta)} \mathscr{K}_{\zeta} .
$$

Proof. It suffices to recall that for $h \in A^{2}, \mathscr{P}\left(\bar{h} \mathscr{K}_{\zeta}\right)=\bar{h}(\zeta) \mathscr{K}_{\zeta}$.

The next lemma is a generalization of Lemma 5 in [1].

LemMA 4.3. Let $f \in \mathscr{H}(B), j$ be a positive integer. Let $0<\rho<1$. Then

$$
\int_{B}\left|\Delta_{j} f(w, z)\right|^{2} \frac{\left(1-|w|^{2}\right)^{-\rho}}{|1-z \cdot \bar{w}|^{n+1}} d m(w) \leq c\left(1-|z|^{2}\right)^{-\rho}\|f\|_{\mathscr{B}}^{2} .
$$

Proof. Write $F(w, z)$ for $\Delta_{j} f(w, z)$. Using the identities in [14], 2.2.2 and the change of variables $w=\varphi_{z}(\zeta)$, we obtain

$$
\begin{aligned}
\int_{B}|F(w, z)|^{2} \frac{\left(1-|w|^{2}\right)^{-\rho}}{|1-z \cdot \bar{w}|^{n+1}} d m(w) \\
\quad=\int_{B}\left|F\left(\varphi_{z}(\zeta)\right), z\right|^{2} \frac{\left(1-\left|\varphi_{z}(\zeta)\right|^{2}\right)^{-\rho}}{\left|1-z \cdot \bar{\varphi}_{z}(\zeta)\right|^{n+1}} \frac{\left(1-|z|^{2}\right)^{n+1}}{|1-\zeta \cdot \bar{z}|^{2(n+1)}} d m(\zeta) \\
\quad=\left(1-|z|^{2}\right)^{-\rho} \int_{B}\left|F\left(\varphi_{z}(\zeta), z\right)\right|^{2} \frac{\left(1-|\zeta|^{2}\right)^{-\rho}}{|1-\zeta \cdot \bar{z}|^{n+1-2 \rho}} d m(\zeta)
\end{aligned}
$$

Let

$$
1<q^{\prime}<\min \left(\frac{1}{\rho}, \frac{n+1}{n+1-\rho}\right),
$$

and set $q=q^{\prime} /\left(q^{\prime}-1\right)$. Then applying Hölder's inequality to equation (10) we obtain

$$
\begin{aligned}
\int_{B}|F(w, z)|^{2} \frac{\left(1-|w|^{2}\right)^{-\rho}}{|1-z \cdot \bar{w}|^{n+1}} d m(w) \\
\leq\left(1-|z|^{2}\right)^{-\rho}\left(\int_{B}\left|F\left(\varphi_{z}(\zeta), z\right)\right|^{2 q} d m(\zeta)\right)^{1 / q} \\
\quad \times\left(\int_{B} \frac{\left(1-|\zeta|^{2}\right)^{-\rho q^{\prime}}}{|1-\zeta \cdot \bar{z}|^{(n+1-2 \rho) q^{\prime}}} d m(\zeta)\right)^{1 / q^{\prime}}
\end{aligned}
$$

Because of our choice of $q^{\prime}$, it follows that $-\rho q^{\prime}>-1$ and $(n+1-2 \rho) q^{\prime}<n+1-\rho q^{\prime}$. Now, 2.4 implies that the second integral in the above inequality is bounded by a constant. Therefore applying 3.4 we obtain the result.

Now we are ready to prove one of the main results of the paper. 
THEOREM 4.4. Let $f \in \mathscr{H}(B), j$ be any positive integer. Let $k_{\zeta}$ be the normalized reproducing kernel; i.e. $\mathscr{K}_{\zeta} /\left\|\mathscr{K}_{\zeta}\right\|$. Then the following quantities are equivalent.

$$
\begin{aligned}
\mathrm{I} & :=\sup _{\zeta \in B}\left(1-|\zeta|^{2}\right)^{j}\left|R^{j} f(\zeta)\right| . \\
\mathrm{II}^{\prime} & :=\sup _{\zeta \in B}\left\|\Delta_{j} f\left(\zeta, \varphi_{\zeta}(\cdot)\right)\right\|_{L^{2}(d m)} . \\
\mathrm{III} & :=\sup _{\zeta \in B}\left\|H_{f ; j} k_{\zeta}\right\| \cdot \\
\mathrm{IV} & :=\left\|H_{f ; j}\right\| .
\end{aligned}
$$

Proof. By 3.4 we know that

$$
\mathrm{I} \lesssim \mathrm{II}^{\prime}
$$

By 4.2 it follows that

$$
\left|H_{f ; j} k_{\zeta}(z)\right|=\left|\Delta_{j} f(\zeta, z) k_{\zeta}(z)\right|
$$

so that

$$
\begin{aligned}
\left\|H_{f ; j} k_{\zeta}\right\|^{2} & =\int_{B}\left|\Delta_{j} f(\zeta, z)\right|^{2}\left|k_{\zeta}\right|^{2} d m(z) \\
& =\left\|\Delta_{j} f\left(\zeta, \varphi_{\zeta}(\cdot)\right)\right\|_{L^{2}(d m)}^{2},
\end{aligned}
$$

i.e.

$$
\mathrm{II}^{\prime}=\text { III . }
$$

It is trivial that $\mathrm{III} \leq \mathrm{IV}$. We now prove that IV $\lesssim \mathrm{I}$. Let $g \in A^{2}$. Then applying 4.3 we obtain

$$
\begin{aligned}
\left|H_{f ; j} g(z)\right|^{2} & \\
\leq & \left(\int_{B}\left|\Delta_{j} f(w, z)\right| \frac{|g(w)|}{|1-z \cdot \bar{w}|^{n+1}} d m(w)\right)^{2} \\
\leq & \int_{B}\left|\Delta_{j} f(w, z)\right|^{2} \frac{\left(1-|w|^{2}\right)^{-\rho}}{|1-z \cdot \bar{w}|^{n+1}} d m(w) \\
& \times \int_{B}|g(w)|^{2} \frac{\left(1-|w|^{2}\right)^{\rho}}{|1-z \cdot \bar{w}|^{n+1}} d m(w) \\
\leq & c\left(1-|z|^{2}\right)^{-\rho}\|f\|_{\mathscr{B}}^{2} \int_{B}|g(z)|^{2} \frac{\left(1-|w|^{2}\right)^{\rho}}{|1-z \cdot \bar{w}|^{n+1}} d m(w) .
\end{aligned}
$$

Thus,

$$
\begin{aligned}
\left\|H_{f ; j} g\right\|^{2} & \leq\|f\|_{\mathscr{B}}^{2} \int_{B}\left(1-|w|^{2}\right)^{\rho}|g(w)|^{2} \int_{B} \frac{\left(1-|z|^{2}\right)^{-\rho}}{|1-z \cdot \bar{w}|^{n+1}} d m(z) d m(w) \\
& \leq c\|f\|_{\mathscr{B}}^{2}\|g\|^{2}
\end{aligned}
$$

by 2.4 . 
Corollary 4.5. Let $f \in \mathscr{H}(B), j$ be any positive integer. Then the following are equivalent.

$$
\begin{aligned}
\mathrm{I} & :=\sup _{\zeta \in B}\left(1-|\zeta|^{2}\right)^{j}\left|R^{j} f(\zeta)\right| . \\
\mathrm{II}^{\prime} & :=\sup _{\zeta \in B}\left(\int_{B}\left|\Delta_{j} f\left(\zeta, \varphi_{\zeta}(w)\right)\right|^{2} d m(w)\right)^{1 / 2} .
\end{aligned}
$$

Moreover, let $0<\rho<1$. Then

$$
\int_{B}\left|\Delta_{j} f(z, w)\right|^{2} \frac{\left(1-|w|^{2}\right)^{-\rho}}{|1-z \cdot \bar{w}|^{n+1}} d m(w) \operatorname{lec}\left(1-|z|^{2}\right)^{-\rho}\|f\|_{\mathscr{B}}^{2} .
$$

Proof. The equivalence of II and $\mathrm{II}^{\prime}$ is contained in 4.4. With this, the same proof as 4.3 gives the second part of the statement.

The following is the companion of 4.4 .

THEOREM 4.6. Let $f \in \mathscr{H}(B), j$ be any positive integer. Then the following are equivalent.

$$
\begin{aligned}
\mathrm{I} & :=\sup _{\zeta \in B}\left(1-|\zeta|^{2}\right)^{j}\left|R^{j} f(\zeta)\right| . \\
\mathrm{II} & :=\sup _{\zeta \in B}\left\|\Delta_{j} f\left(\varphi_{\zeta}(\cdot), \zeta\right)\right\|_{L^{2}(d m)} . \\
\mathrm{III}^{\prime} & :=\sup _{\zeta \in B}\left\|H_{f ; j}^{\prime} k_{\zeta}\right\| . \\
\mathrm{IV}^{\prime} & :=\left\|H_{f ; j}^{\prime}\right\| .
\end{aligned}
$$

Proof. As in the previous proof we can see that $\mathrm{II}=\mathrm{III}^{\prime}$. Thus, we know that

$$
\mathrm{I} \leq \mathrm{II}=\mathrm{III}^{\prime} \leq \mathrm{IV}^{\prime} .
$$

Now, that $I V^{\prime} \leq \mathrm{I}$ follows from 4.3 .

Proof of 1.7. It follows immediately from 4.4 and 4.6.

Proof of 1.8. Let $f \in \mathscr{B}_{0}$. Write $T$ for any of the generalized Hankel operators $H_{f ; j}$ and $H_{f ; j}^{\prime}$, and $F(z, w)$ for either $-\Delta_{j} f(w, z)$ or $\Delta_{j} f(z, w)$. Let $0<\rho<1$. For $g \in A^{2}$ we have

$$
\begin{aligned}
T g(z)= & \chi_{\{|z| \leq \rho\}} \int_{B} \overline{F(z, w)} \mathscr{K}(z, w) d m(w) \\
& +\chi_{\{\rho<|z|<1\}} \int_{B} \overline{F(z, w)} \mathscr{K}(z, w) d m(w) \\
\equiv & T_{1} g(z)+T_{2} g(z) .
\end{aligned}
$$


We first show that $T_{1}$ is compact. Let $\left\{g_{l}\right\}$ be a sequence weakly converging to 0 . Then,

$$
\begin{aligned}
\left|T_{1} g_{l}\right|^{2} \leq & \int_{|z| \leq \rho} \int_{B}\left|F\left(\varphi_{z}(w), z\right)\right|^{2}|\mathscr{K}(z, w)|\left(1-|w|^{2}\right)^{-\varepsilon} d m(w) \\
& \times \int_{B}\left|g_{l}(w)\right|^{2}|\mathscr{K}(z, w)|\left(1-|w|^{2}\right)^{\varepsilon} d m(w) d m(z) \\
\leq & c\left(1-\rho^{2}\right)^{-\varepsilon}\|f\|_{\mathscr{B}} \int_{B}\left|g_{l}(w)\right|^{2}\left(1-|w|^{2}\right)^{-\varepsilon} \log (1-|w|)^{2} d m(w),
\end{aligned}
$$

which tends to 0 as $l$ tends to $\infty$. Next, by the same argument as in 4.3 it follows that for any given $\varepsilon>0$ we can choose $\rho$ close enough to 1 so that

$$
\left\|T_{2}\right\| \leq \sup _{\rho<\zeta<1} \widetilde{D} f(\zeta)^{2} \leq c \varepsilon .
$$

Since $T$ can be approximated by compact operators in norm, it follows that $T$ is compact.

If $T$ is compact then $\left\|T k_{\zeta}\right\| \rightarrow 0$ as $|\zeta| \rightarrow 1^{-}$, which implies that $f \in \mathscr{B}_{0}$ by 3.5 and 4.5 . This concludes the proof.

We now turn to the Schatten-ideal properties of the generalized Hankel operators. It turns out that the cut-off depends in an essential way on the order of zero of (the expression defined by) the symbol $f$. We remark that our result contains as particular case (when $j=1$ ) the unweighted case of the theorems in [2], [7], [19], and [21].

Proof of 1.9. Write $T_{j}$ to indicate any of the generalized Hankel operators $H_{f ; j}$ and $H_{f ; j}^{\prime}$. By 1.2 it follows that

$$
\left\|T_{j}\right\|_{S_{2}} \approx\|f\|_{B_{2}}
$$

for $j>n$. For $j \leq n$, if $\left\|T_{j}\right\|_{B_{2}}$ is finite, then $f$ is a polynomial of degree at most $j-1$, i.e. $T_{j}=0$.

Let $2<p<\infty$. We use Lemma 3.6 of [2], which is an improvement of a result of Russo, [15]. Let $j>2 n / p$. Then,

$$
\int_{B} \int_{B}\left|\Delta_{j} f(w, z)\right|^{p}|\mathscr{K}(z, w)|^{2} d m(z) d m(w) \leq c\|f\|_{B_{p}}^{p}<\infty
$$

implies that $T_{j} \in S_{p}$ with $S_{p}$-norm bounded by the $B_{p}$-norm of $f$.

Conversely, if $T$ is any bounded operator from $A^{2}$ into a Hilbert space $H$, one can see that 


$$
\|T\|_{S_{2}}^{2}=\int_{B}\left\|T \mathscr{K}_{\zeta}\right\|_{H}^{2} d m(\zeta)=\int_{B}\left\|T k_{\zeta}\right\|_{H}^{2} d \lambda(\zeta) .
$$

Using interpolation with the obvious case $p=\infty$ it follows that for $2 \leq p \leq \infty$, and $T \in S_{p}$,

$$
\|\| T k_{\zeta}\left\|_{H}\right\|_{L^{p}(d \lambda)} \leq\|T\|_{S_{p}} .
$$

Next, let $T_{j}$ be either $H_{f ; j}$ or $H_{f ; j}^{\prime}$. By $4.2\left\|T_{j} k_{\zeta}\right\|=\left\|F(\zeta, \cdot) k_{\zeta}\right\|_{A_{2}}$, where $F$ means the appropriate expression between $\Delta_{j} f(w, \zeta)$ and $\Delta_{j} f(\zeta, w)$. Now we apply 3.3 with $q=2$. It follows that

$$
\left\|\Delta_{j} f\right\|_{L^{p}(d \Lambda)} \leq\left\|T_{j}\right\|_{S_{p}} .
$$

This implies that if $T \in S_{p}$ and $j>2 n / p$, then $f \in B_{p}$. If $T \in S_{p}$ and $j \leq 2 n / p$, then $f$ is a polynomial of degree less than $j$ (and $T_{j}=0$ ) as a consequence of 3.3. again.

REMARK. It is the author's belief that the result of 1.9 should hold for the range $0<p<2$ too. In this case a more detailed analysis seems to be needed.

5. Proof of 3.1. In this section we prove 3.1. The proof of the case $j=1$, using 2.2 and the Möbius invariance, does not give the general result. Hence, a different approach is needed. We split the proof in the cases $0<p \leq 1$ and $1<p<\infty$. When $0<p \leq 1$ we use the atomic decomposition of the Besov spaces. For the case $1<p<\infty$ we use an integration by parts and Hölder's inequality. However, in both cases we need an estimate of Forelli-Rudin type (see 2.4) for certain integrals. These integrals come from the expression for $\Delta_{j} K_{\zeta}$ where $K_{\zeta}$ is a suitable power of the reproducing kernel.

LEMMA 5.1. Let $j, \nu$ be positive integers, and $\zeta \in B$. Let $K_{\zeta}$ be defined by

$$
K_{\zeta}(z)=\frac{1}{(1-z \cdot \bar{\zeta})^{\nu}}
$$

Moreover, let

$$
M_{i}(w, z, \zeta)=\frac{((z-w) \cdot \bar{\zeta})^{j+i}}{(1-w \cdot \bar{\zeta})^{\nu}(1-z \cdot \bar{\zeta})^{j+i}}
$$

Then

$$
\Delta_{j} K_{\zeta}(w, z)=\sum_{i=0}^{\nu-1} d_{i} M_{i}(w, z, \zeta),
$$

for some positive constants $d_{i}$. 
Proof. By rotational invariance it suffices to prove the lemma for $\zeta=\zeta_{1} e_{1}$, where $e_{1}$ is the base vector $(1,0, \ldots, 0)$. Write $g$ for $K_{\zeta_{1} e_{1}}$. We first show that

(11) $\Delta_{j} K_{\zeta}(w, z)$

$$
\begin{aligned}
= & \frac{1}{\left(1-w_{1} \bar{\zeta}_{1}\right)^{\nu}\left(1-z_{1} \bar{\zeta}_{1}\right)^{\nu+j-1}} \\
& \times\left(\left(1-z_{1} \bar{\zeta}_{1}\right)^{\nu+j-1}\right. \\
& \left.\quad-\left.\sum_{i=0}^{j-1} \frac{1}{i !}\left(\frac{d}{d z_{1}}\right)^{i}\left(1-z_{1} \bar{\zeta}_{1}\right)^{\nu+j-1}\right|_{z_{1}=w_{1}}\left(w_{1}-z_{1}\right)^{i}\right) .
\end{aligned}
$$

Indeed we have,

(12) $\Delta_{j} K_{\zeta}(w, z)$

$$
\begin{aligned}
& =\frac{1}{\left(1-z_{1} \bar{\zeta}_{1}\right)^{\nu+j-1}} \\
& \times\left(\frac{\left(1-z_{1} \bar{\zeta}_{1}\right)^{\nu+j-1}}{\left(1-w_{1} \bar{\zeta}_{1}\right)^{\nu}}\right. \\
& \left.-\sum_{i=0}^{j-1}\left(\begin{array}{c}
\nu+i-1 \\
i
\end{array}\right) \bar{\zeta}_{1}^{i}\left(w_{1}-z_{1}\right)^{i}\left(1-z_{1} \bar{\zeta}_{1}\right)^{j-i-1}\right) \text {. }
\end{aligned}
$$

Now write

$$
\begin{aligned}
& \left(1-z_{1}\right)^{j-i-1} \\
& \quad=\left(\left(1-w_{1} \bar{\zeta}_{1}\right)-\left(w_{1}-z_{1}\right) \bar{\zeta}_{1}\right)^{j-i-1} \\
& \quad=\sum_{l=0}^{j-i-1}\left(\begin{array}{c}
j-i-1 \\
l
\end{array}\right)\left(1-w_{1} \bar{\zeta}_{1}\right)^{i}\left(\left(w_{1}-z_{1}\right) \bar{\zeta}_{1}\right)^{j-i-1-l}
\end{aligned}
$$

and substitute (13) into (12). Using the identity

$$
\sum_{l=0}^{j-i-1}\left(\begin{array}{c}
\nu+l-1 \\
l
\end{array}\right)\left(\begin{array}{c}
j-l-1 \\
i
\end{array}\right)=\left(\begin{array}{c}
\nu+j-1 \\
j-i-1
\end{array}\right)
$$

we obtain (11). Finally, 


$$
\begin{gathered}
\left(1-z_{1} \bar{\zeta}_{1}\right)_{i=0}^{\nu+j-1}-\sum_{i=0}^{j-1}\left(\begin{array}{c}
\nu+j-1 \\
i
\end{array}\right)\left(1-w_{1} \bar{\zeta}_{1}\right)^{\nu+j-1-i}\left(\left(w_{1}-z_{1}\right) \bar{\zeta}_{1}\right)^{i} \\
=\left(\left(w_{1}-z_{1}\right) \bar{\zeta}_{1}\right)^{j} \sum_{i=0}^{\nu-1} c_{i}\left(1-w_{1} \bar{\zeta}_{1}\right)^{\nu-1-i}\left(\left(w_{1}-z_{1}\right) \bar{\zeta}_{1}\right)^{i} \\
=\left(\left(w_{1}-z_{1}\right) \bar{\zeta}_{1}\right)^{j} \sum_{l=0}^{\nu-1} d_{l}\left(1-z_{1} \bar{\zeta}_{1}\right)^{\nu-1-l}\left(\left(w_{1}-z_{1}\right) \bar{\zeta}_{1}\right)^{l}
\end{gathered}
$$

This, together with (11), concludes the proof.

LeMmA 5.2. Let $n \geq 1$. Let $d>2 n$ if $n>1$, and $d>1$ if $n=1$. Let $0 \leq \varepsilon<1$. Then there exists an absolute constant $C_{0}$ such that

$$
\begin{aligned}
\int_{B} \int_{B} & \frac{\left|\left(z_{1}-w_{1}\right) \bar{\zeta}_{1}\right|^{d}}{\left|1-z_{1} \bar{\zeta}_{1}\right|^{d}\left|1-w_{1} \bar{\zeta}_{1}\right|^{d}} \frac{\left(1-\left|z_{1}\right|^{2}\right)^{-\varepsilon}\left(1-\left|w_{1}\right|^{2}\right)^{-\varepsilon}}{\left|1-z_{1} \bar{w}_{1}\right|^{2(n+1-\varepsilon)}} d m(z) d m(w) \\
& \leq \frac{C_{0}}{\left(1-\left|\zeta_{1}\right|^{2}\right)^{d}}
\end{aligned}
$$

Proof. It suffices to notice that, if $\varphi_{\zeta_{1}} \equiv \varphi_{\zeta_{1} e_{1}}$ is defined as in (3), and $v_{1}(z)=z_{1}$ is the coordinate function, then

$$
\frac{\left|\left(z_{1}-w_{1}\right)\right|\left(1-\left|\zeta_{1}\right|^{2}\right)}{\left|1-w_{1} \bar{\zeta}_{1}\right|\left|1-z_{1} \bar{\zeta}_{1}\right|}=\left|v_{1} \circ \varphi_{\zeta_{1}}(w)-v_{1} \circ \varphi_{\zeta_{1}}(z)\right|
$$

Then applying [3] Theorem 1, when $n>1$, or the results in [4] or in [23] when $n=1$, we obtain that

$$
\begin{aligned}
& \int_{B} \int_{B} \frac{\left|\left(z_{1}-w_{1}\right) \bar{\zeta}_{1}\right|^{d}}{\left|1-z_{1} \bar{\zeta}_{1}\right|^{d}\left|1-w_{1} \bar{\zeta}_{1}\right|^{d}} \frac{\left(1-\left|z_{1}\right|^{2}\right)^{-\varepsilon}\left(1-\left|w_{1}\right|^{2}\right)^{-\varepsilon}}{\left|1-z_{1} \bar{w}_{1}\right|^{2(n+1-\varepsilon)}} d m(z) d m(w) \\
& \quad \leq \frac{C}{\left(1-\left|\zeta_{1}\right|^{2}\right)^{d}} \int_{B} \int_{B}\left|v_{1} \circ \varphi_{\zeta_{1} e_{1}}(z)-v_{1} \circ \varphi_{\zeta_{1}, e_{1}}(w)\right|^{d} \\
& \quad \times \frac{\left(1-\left|z_{1}\right|^{2}\right)^{-\varepsilon}\left(1-\left|w_{1}\right|^{2}\right)^{-\varepsilon}}{\left|1-z_{1} \bar{w}_{1}\right|^{2(n+1-\varepsilon)}} d m(z) d m(w) \\
& \quad \leq C \frac{\left\|v_{1}\right\|_{B_{d}}^{d}}{\left(1-\left|\zeta_{1}\right|^{2}\right)^{d}},
\end{aligned}
$$

where $C$ in (another) constant, independent of $\zeta$. 
LEMmA 5.3. Let $f \in \mathscr{H}(B)$. Let $1 \leq p<\infty$. Then there exists $c_{0}>0$ such that for $0 \leq \varepsilon \leq \varepsilon_{0}$

$$
\begin{gathered}
\int_{B} \int_{B}\left|f \circ \varphi_{\zeta_{1} e_{1}}(z)-f \circ \varphi_{\zeta_{1} e_{1}}(w)\right|^{p} \frac{\left(1-|w|^{2}\right)^{-\varepsilon}}{\left|1-z_{1} \bar{w}_{1}\right|^{2(n+1)}} d m(z) d m(w) \\
\leq \frac{C}{\left(1-|\zeta|^{2}\right)^{\varepsilon}} \int_{B}|\widetilde{D} f(z)|^{p}\left(1-|z|^{2}\right)^{-(n+1+\varepsilon)} d m(z) .
\end{gathered}
$$

REMARK. Notice that this is an extension to the case $\varepsilon>0$ of one of the equivalences contained in [9] Theorem 1 . Also recall that $\widetilde{D} f$ is the invariant derivative introduced in (5).

Proof. We change the variable $z=\varphi_{w}(\xi)$ in the first integral. Recall the formulas 2.2.2 and 2.2.6 in [14]. We also use 2.4, and Lemma 3.2 and Remark 3.2 of [3]. We obtain

$$
\begin{aligned}
\int_{B} \int_{B}\left|f \circ \varphi_{\zeta}(z)-f \circ \varphi_{\zeta}(w)\right|^{p} \frac{d m(z)}{|1-z \cdot \bar{w}|^{2(n+1)}}\left(1-|w|^{2}\right)^{-\varepsilon} d m(w) \\
\quad=\int_{B} \int_{B}\left|f \circ \varphi_{\zeta} \circ \varphi_{w}(\xi)-f \circ \varphi_{\zeta} \circ \varphi_{w}(0)\right|^{p} d m(\xi) \\
\quad \leq C \int_{B} \int_{B}\left|(\widetilde{D} f)\left(\varphi_{\varphi_{\zeta}(w)}(\xi)\right)\right|^{p} d m(\xi)\left(1-|w|^{2}\right)^{-(n+1+\varepsilon)} d m(w) \\
\quad=\int_{B} \int_{B}|(\widetilde{D} f)(z)|^{p} \frac{\left(1-\left|\varphi_{\zeta}(w)\right|^{2}\right)^{n+1}}{\left|1-z \cdot \bar{\varphi}_{\zeta}(w)\right|^{2(n+1)} d m(z)} \\
\quad=\int_{B}|(\widetilde{D} f)(z)|^{p} \int_{B} \frac{\left(1-|\eta|^{2}\right)^{n+1}\left(1-\left|\varphi_{\zeta}(\eta)\right|^{2}\right)^{-(n+1+\varepsilon)}}{|1-z \cdot \bar{\eta}|^{2(n+1)}} d m(w) \\
\quad=\int_{B}|(\widetilde{D} f)(z)|^{p} \int_{B} \frac{\left(1-|\eta|^{2}\right)^{-\varepsilon}\left(1-|\zeta|^{2}\right)^{-\varepsilon}}{|1-\zeta \cdot \bar{\eta}|^{-2 \varepsilon}} d m(\eta) \frac{\left(\left.\zeta\right|^{2}\right)^{n+1}}{\mid 1-\eta(n)} d m(\eta) d m(z) \\
\quad \leq \frac{1}{\left(1-|\zeta|^{2}\right)^{\varepsilon}} \int_{B}|(\widetilde{D} f)(z)|^{p} \int_{B} \frac{\left(1-|\eta|^{2}\right)^{-\varepsilon}}{|1-\eta \cdot \bar{\zeta}|^{2(n+1)}} d m(\eta) d m(z) \\
\quad \leq \frac{C}{\left(1-|\zeta|^{2(n+1)}\right.} \int_{B}|(\widetilde{D} f)(z)|^{p}\left(1-|z|^{2}\right)^{-(n+1+\varepsilon)} d m(z) .
\end{aligned}
$$

Proposition 5.4. Let $c(n)=2 n$ if $n>1$, and $c(n)=1$ if $n=1$. Let $a, b \in \mathbf{R}, a, b>c(n)$. Let $0 \leq 2 \varepsilon<\min (a, b)-c(n)$. Then 
there exists an absolute constant $C_{0}$ such that

$$
\begin{aligned}
& \int_{B} \int_{B} \frac{|(z-w) \cdot \bar{\zeta}|^{a}}{|1-w \cdot \bar{\zeta}|^{b}|1-z \cdot \bar{\zeta}|^{a}} \frac{\left(1-|w|^{2}\right)^{-\varepsilon}}{|1-z \cdot \bar{w}|^{2(n+1)}} d m(z) d m(w) \\
& \quad \leq \frac{C_{0}}{\left(1-|\zeta|^{2}\right)^{b+\varepsilon}}
\end{aligned}
$$

Proof. We give the proof in the case $n>1$. If $n=1$ the proof follows the same pattern as in the case $n>1$, but some step has to be considered void. We leave the details to the reader.

By rotational invariance we may assume that $\zeta=\zeta_{1} e_{1}$ where $e_{1}=$ $(1,0, \ldots, 0)$ is the base vector. We break the integral into two over the regions $\Omega$ and $\Omega^{\prime}$. The region $\Omega$ is defined by

$$
\Omega=\left\{(z, w) \in B \times B:\left|z_{1}-w_{1}\right| \leq \frac{1}{4}\left(\left(1-\left|z_{1}\right|^{2}\right)+\left(1-\left|w_{1}\right|^{2}\right)\right)\right\} .
$$

Notice that in this case

$$
\left|1-z_{1} \bar{\zeta}_{1}\right| \leq 3\left|1-w_{1} \bar{\zeta}_{1}\right| \leq 9\left|1-z_{1} \bar{\zeta}_{1}\right|
$$

Thus, if $d=\min (a, b)$,

$$
\frac{|(z-w) \cdot \bar{\zeta}|^{a}}{|1-w \cdot \bar{\zeta}|^{b}|1-z \cdot \bar{\zeta}|^{a}} \leq \frac{C}{\left(1-|\zeta|^{2}\right)^{b-d}} \frac{|(z-w) \cdot \bar{\zeta}|^{d}}{|1-w \cdot \bar{\zeta}|^{d}|1-z \cdot \bar{\zeta}|^{d}} .
$$

Let $v_{1}$ be the coordinate function $v_{1}(z)=z_{1}$. Thus, the integral that we want to estimate is less or equal to a constant times

$$
\begin{aligned}
\frac{1}{\left(1-\left|\zeta_{1}\right|^{2}\right)^{b}} \int_{B} \int_{B}\left|v_{1} \circ \varphi_{\zeta_{1} e_{1}}(z)-v_{1} \circ \varphi_{\zeta_{1} e_{1}}(w)\right|^{d} \\
\times \frac{\left(1-|w|^{2}\right)^{-\varepsilon}}{|1-z \cdot \bar{w}|^{2(n+1)}} d m(z) d m(w) .
\end{aligned}
$$

Now we apply 5.3. Because of our choice of $d$ and $\varepsilon$ the integral

$$
\int_{B}\left|\widetilde{D} v_{1}\right|^{p}\left(1-|z|^{2}\right)^{-(n+1+\varepsilon)} d m(z)
$$

is finite. This gives the estimate for $\Omega$.

The region $\Omega^{\prime}$ is defined by

$$
\Omega^{\prime}=\left\{(z, w) \in B \times B:\left|z_{1}-w_{1}\right| \geq \frac{1}{4}\left(\left(1-\left|z_{1}\right|^{2}\right)+\left(1-\left|w_{1}\right|^{2}\right)\right)\right\} .
$$


Write $z=\left(z_{1}, z^{\prime}\right)$ and $w=\left(w_{1}, w^{\prime}\right)$. Now we integrate out.

$$
\begin{aligned}
\iint_{\Omega^{\prime}} & \frac{|(z-w) \cdot \bar{\zeta}|^{a}}{|1-w \cdot \bar{\zeta}|^{b}|1-z \cdot \bar{\zeta}|^{a}} \frac{\left(1-|w|^{2}\right)^{-\varepsilon}}{|1-z \cdot \bar{w}|^{2(n+1)}} d m(z) d m(w) \\
\leq \iint_{\Omega^{\prime} \cap D \times D} & \frac{|(z-w) \cdot \bar{\zeta}|^{a}}{|1-w \cdot \bar{\zeta}|^{b}|1-z \cdot \bar{\zeta}|^{a}} \int_{\left|z^{\prime}\right|<\left(1-\left|z_{1}\right|^{2}\right)^{1 / 2}} \int_{\left|w^{\prime}\right|<\left(1-\left|w_{1}\right|^{2}\right)^{1 / 2}} \\
& \times \frac{\left(1-|w|^{2}\right)^{-\varepsilon}}{|1-z \cdot \bar{w}|^{2(n+1)}} d m\left(w^{\prime}\right) d m\left(z^{\prime}\right) d m\left(w_{1}\right) d m\left(z_{1}\right) .
\end{aligned}
$$

Consider the two inner integrals:

$$
\begin{array}{r}
\int_{\left|z^{\prime}\right|<\left(1-\left|z_{1}\right|^{2}\right)^{1 / 2}} \int_{\left|w^{\prime}\right|<\left(1-\left|w_{1}\right|^{2}\right)^{1 / 2}} \frac{\left(1-|w|^{2}\right)^{-\varepsilon}}{|1-z \cdot \bar{w}|^{2(n+1)}} d m\left(w^{\prime}\right) d m\left(z^{\prime}\right) \\
=\int_{\left|z^{\prime}\right|<\left(1-\left|z_{1}\right|^{2}\right)^{1 / 2}} \int_{\left|w^{\prime}\right|<\left(1-\left|w_{1}\right|^{2}\right)^{1 / 2}} \frac{\left(\left(1-\left|w_{1}\right|^{2}\right)-\left|w^{\prime}\right|^{2}\right)^{-\varepsilon}}{\left|1-z_{1} \bar{w}_{1}-z^{\prime} \cdot \bar{w}^{\prime}\right|^{2(n+1)}} \\
\times d m\left(w^{\prime}\right) d m\left(z^{\prime}\right) .
\end{array}
$$

Now we perform the change of variables:

$$
u=\frac{z^{\prime}}{\left(1-\left|z_{1}\right|^{2}\right)^{1 / 2}}, \quad \text { and } \quad v=\frac{w^{\prime}}{\left(1-\left|w_{1}\right|^{2}\right)^{1 / 2}} .
$$

Thus, the above integrals equal

$$
\begin{aligned}
& \frac{\left(1-\left|z_{1}\right|^{2}\right)^{n-1}\left(1-\left|w_{1}\right|^{2}\right)^{n-1-\varepsilon}}{\left|1-z_{1} \bar{w}_{1}\right|^{2(n+1)}} \\
& \quad \times \int_{|u|<1} \int_{|v|<1} \frac{\left(1-|v|^{2}\right)^{-\varepsilon}}{|1-Q u \cdot \bar{v}|^{2(n+1)}} d m(u) d m(v),
\end{aligned}
$$

where we have set

$$
Q=\frac{\left(1-\left|z_{1}\right|^{2}\right)^{1 / 2}\left(1-\left|w_{1}\right|^{2}\right)^{1 / 2}}{\left(1-z_{1} \bar{w}_{1}\right)}
$$

Next, 2.4 gives (recall that $u, v \in \mathbf{C}^{n-1}$ )

$$
\begin{aligned}
& \int_{|u|<1} \int_{|v|<1} \frac{\left(1-|v|^{2}\right)^{-\varepsilon}}{|1-Q u \cdot \bar{v}|^{2(n+1)}} d m(u) d m(v) \\
& \quad \leq \int_{|u|<1} \frac{1}{\left(1-|Q u|^{2}\right)^{n+2+\varepsilon}} d m(u) \leq \frac{C}{\left(1-|Q|^{2}\right)^{n+1+\varepsilon}} .
\end{aligned}
$$

Now notice that, since $(z, w) \in \Omega^{\prime}$,

$$
\frac{1}{\left(1-|Q|^{2}\right)}=\frac{\left|1-z_{1} \bar{w}_{1}\right|^{2}}{\left|z_{1}-w_{1}\right|^{2}}<25 \text {. }
$$


Therefore, the integral over the region $\Omega^{\prime}$ is less or equal to a constant times

$$
\begin{aligned}
\int_{D} \int_{D} & \frac{\left|\left(z_{1}-w_{1}\right) \cdot \bar{\zeta}_{1}\right|^{a}}{\left|1-w_{1} \bar{\zeta}_{1}\right|^{b}\left|1-z_{1} \bar{\zeta}_{1}\right|^{a}} \\
& \times \frac{\left(1-\left|z_{1}\right|^{2}\right)^{n-1}\left(1-\left|w_{1}\right|^{2}\right)^{n-1-\varepsilon}}{\left|1-z_{1} \bar{w}_{1}\right|^{2(n+1)}} d m\left(z_{1}\right) d m\left(w_{1}\right) \\
\leq & \frac{C}{\left(1-\left|\zeta_{1}\right|^{2}\right)^{b-d}} \int_{D} \int_{D} \frac{\left|\left(z_{1}-w_{1}\right) \cdot \bar{\zeta}_{1}\right|^{d}}{\left|1-w_{1} \bar{\zeta}_{1}\right|^{d}\left|1-z_{1} \bar{\zeta}_{1}\right|^{d}} \\
& \times \frac{\left(1-\left|w_{1}\right|^{2}\right)^{-\varepsilon}}{\left|1-z_{1} \bar{w}_{1}\right|^{4}} d m\left(z_{1}\right) d m\left(w_{1}\right),
\end{aligned}
$$

where $d=\min (a, b)$. Now we apply 5.3 with $f(z)=z_{1}$. This finishes the proof.

Proof of 3.1. Case $0<p \leq 1$. This follows easily from the atomic decomposition and 5.4. Let $f \in B_{p}$, let $\left\{\zeta^{i}\right\}$ be a $\vartheta$-lattice. Then there exist constants $\left\{c_{i}\right\}$ such that

$$
f(z)=\sum_{i=1}^{\infty} c_{i} \frac{\left(1-\left|\zeta^{i}\right|^{2}\right)^{\nu}}{\left(1-z \cdot \bar{\zeta}_{i}\right)^{\nu}}
$$

and

$$
\|f\|_{B_{p}}^{p} \approx \sum\left|c_{i}\right|^{p}
$$

Hence,

$$
\begin{aligned}
\left|\Delta_{j} f(w, z)\right|^{p} & \leq\left(\sum\left|c_{i}\right|\left(1-|\zeta|^{2}\right)^{\nu}\left|\Delta_{j} K_{\zeta^{i}}(w, z)\right|\right)^{p} \\
& \leq \sum\left|c_{i}\right|^{p}\left(1-\left|\zeta^{i}\right|^{2}\right)^{\nu p}\left|\Delta_{j} K_{\zeta^{i}}\right|^{p} .
\end{aligned}
$$

Therefore, for $j>2 n / p$, by 5.4 (with $\varepsilon=0$ ), it follows that

$$
\begin{aligned}
\left\|\Delta_{j} f\right\|_{L^{p}(d \Lambda)}^{p} & \leq \sum\left|c_{i}\right|^{p}\left\|\Delta_{j} K_{\zeta^{i}}\right\|_{L^{p}(d \Lambda)}^{p}\left(1-\left|\zeta^{i}\right|^{2}\right)^{\nu p} \\
& \leq C_{0} \sum\left|c_{i}\right|^{p} \leq C_{0}\|f\|_{B_{p}}^{p} .
\end{aligned}
$$

Case $1<p<\infty$. In this case we will use an integration by parts and Hölder's inequality. Given $p$, let $j$ and $\nu$ be integers satisfying the following conditions. We let $j>2 n / p$. Pick $\varepsilon, 0<\varepsilon<$ $(j p-2 n)(p-1)$. Finally, let $2 n<\nu<2(n+1)+\varepsilon$.

Recall the notation introduced in 5.1. Notice that for $f \in \mathscr{H}(B)$ and $K_{\zeta}=(1-z \cdot \bar{\zeta})^{-\nu}$, the weighted reproducing kernels (see [14] 
7.1.1) give

$$
\begin{aligned}
\Delta_{j} f(w, z) & =c_{\nu} \int_{B} f(\zeta) \Delta_{j} K_{\zeta}(w, z)\left(1-|\zeta|^{2}\right)^{\nu-n-1} d m(\zeta) \\
& =c_{\nu} \sum_{i=0}^{\nu-1} \int_{B} f(\zeta) M_{i}(w, z, \zeta)\left(1-|\zeta|^{2}\right)^{\nu-n-1} d m(\zeta) .
\end{aligned}
$$

Now let $\mathscr{R}_{n+1}^{-m}$ be the integral operator defined for $h \in \mathscr{H}(B)$ by

$$
\mathscr{R}_{n+1}^{-m} h(z)=c_{n+1} \int_{B} \frac{h(w)}{(1-z \cdot \bar{w})^{2(n+1)}}\left(1-|w|^{2}\right)^{n+1+m} d m(w)
$$

Its inverse is the differential operator on $\mathscr{H}(B)$ with constant coefficients (give [12] $\S 3$ for details)

$$
\mathscr{R}_{n+1}^{m} h(z)=c_{m} \int_{B} \frac{h(w)}{(1-z \cdot \bar{w})^{2(n+1)+m}}\left(1-|w|^{2}\right)^{n+1} d m(w)
$$

Here $c_{n+1}$ and $c_{m}$ are suitable positive constants of normalization. Now we apply an integration by parts, using Lemma 1.1 in [5]. Notice that the lemma holds with radial weights since normal derivatives of holomorphic functions can be written in terms of tangential derivatives. Then, applying the integration by parts near the boundary, the derivatives do not fall on the weight. We obtain:

$$
\begin{aligned}
& \int_{B} f(\zeta) M_{i}(z, w, \zeta)\left(1-|\zeta|^{2}\right)^{\nu-n-1} d m(\zeta) \\
&=\int_{B} \mathscr{L}^{m} f(\zeta) \int_{B} \frac{M_{i}(z, w, \eta)\left(1-|\eta|^{2}\right)^{n+1+m}}{(1-\zeta \cdot \bar{\eta})^{2(n+1)}} d m(\eta) \\
& \times\left(1-|\zeta|^{2}\right)^{\nu-n-1} d m(\zeta),
\end{aligned}
$$

where $\mathscr{L}^{m}$ is a differential operator of order $m$ with coefficients $C^{\infty}$ up the boundary. Now notice that, by Fubini's theorem and 2.4, if $n+\varepsilon<\nu<2(n+1)+\varepsilon$,

$$
\left(1-|w|^{2}\right)^{\varepsilon} \int_{B} \int_{B} \frac{\left(1-|\zeta|^{2}\right)^{\nu-n-1-\varepsilon}\left(1-|\eta|^{2}\right)^{n+1}}{|1-w \cdot \bar{\eta}|^{\nu}|1-\zeta \cdot \bar{\eta}|^{2(n+1)}} d m(\zeta) d m(\eta) \leq c_{0}
$$

where $c_{0}$ is a constant independent of $w$. Therefore, applying Jensen 
inequality twice, we obtain

$$
\begin{array}{rl}
\mid \int_{B} & \left.f(\zeta) M_{l}(z, w, \zeta)\left(1-|\zeta|^{2}\right)^{\nu-n-1} d m(\zeta)\right|^{p} \\
\leq & \int_{B}\left|\mathscr{L}^{m} f(\zeta)\right|^{p} \\
& \times\left(\int_{B} \frac{|(z-w) \cdot \bar{\eta}|^{j+l}\left(1-|\eta|^{2}\right)^{n+1+m}\left(1-|\zeta|^{2}\right)^{\nu-n-1}}{|1-z \cdot \bar{\eta}|^{j+i}|1-w \cdot \bar{\eta}|^{\nu}|1-\zeta \cdot \bar{\eta}|^{2(n+1)}} d m(\eta)\right)^{p} d m(\zeta) \\
\leq & C \int_{B}\left|\mathscr{L}^{m} f(\zeta)\right|^{p} \int_{B} \frac{|(z-w) \cdot \bar{\eta}|^{(j+i) p}}{|1-z \cdot \bar{\eta}|^{(j+i) p}} \frac{\left(1-|\eta|^{2}\right)^{m p}}{\left(1-|w|^{2}\right)^{\varepsilon p}}\left(1-|\zeta|^{2}\right)^{\varepsilon p} \\
\leq & \frac{C}{\left(1-|w|^{2}\right)^{\varepsilon(p-1)}} \int_{B}\left|\mathscr{L}^{m} f(\zeta)\right|^{p}\left(1-|\zeta|^{2}\right)^{\varepsilon p+\nu-(n+1+\varepsilon)} \\
& \times \int_{B} \frac{|(z-w) \cdot \bar{\eta}|^{(j+i) p}}{|1-z \cdot \bar{\eta}|^{(j+i) p}} \frac{\left(1-|\eta|^{2}\right)^{n+1+m p}}{|1-w \cdot \bar{\eta}|^{\nu}} \times \frac{1}{|1-\zeta \cdot \bar{\eta}|^{2(n+1)}} d m(\zeta) d m(\zeta) .
\end{array}
$$

Therefore, since $\nu>2 n$,

$\left\|\Delta_{j} f\right\|_{L^{p}(d \Lambda)}^{p}$

$$
\begin{aligned}
\leq & \sum_{i=0}^{\nu-1} \int_{B} \int_{B}\left|\mathscr{L}^{m} f(\zeta)\right|^{p} \frac{\left(1-|\zeta|^{2}\right)^{\varepsilon p+\nu-(n+1+\varepsilon)}\left(1-|\eta|^{2}\right)^{n+1+m p}}{|1-\zeta \cdot \bar{\eta}|^{2(n+1)}} \\
& \times \int_{B} \int_{B} \frac{|(z-w) \cdot \bar{\eta}|^{(j+1) p}}{|1-z \cdot \bar{\eta}|^{(j+1) p}} \frac{\left(1-|w|^{2}\right)^{-\varepsilon(p-1)}}{|1-w \cdot \bar{\eta}|^{\nu}} \\
& \times \frac{d m(z) d m(w)}{|1-z \cdot \bar{w}|^{2(n+1)}} d m(\eta) d m(\zeta) \\
\leq & C \int_{B}\left|\mathscr{L}^{m} f(\zeta)\right|^{p}\left(1-|\zeta|^{2}\right)^{\varepsilon(p-1)+\nu-(n+1)} \\
& \times \int_{B} \frac{\left(1-|\eta|^{2}\right)^{n+1+m p-\nu-\varepsilon(p-1)}}{|1-\zeta \cdot \bar{\eta}|^{2(n+1)}} d m(\eta) d m(\zeta) \\
\leq & C \int_{B}\left|\mathscr{L}^{m} f(\zeta)\right|^{p} \frac{\left(1-|\zeta|^{2}\right)^{\varepsilon(p-1)+\nu-(n+1)}}{\left(1-|\zeta|^{2}\right)^{-m p+\nu+\varepsilon(p-1)}} d m(\zeta),
\end{aligned}
$$

if $m$ is chosen so that $\nu>m p>n$. The last integral is now less or equal to a constant times

$$
\int_{B}\left|R^{m} f(\zeta)\right|^{p}\left(1-|\zeta|^{2}\right)^{m p-(n+1)} d m(\zeta) \equiv\|f\|_{B_{p}}^{p} .
$$

This concludes the proof. 
6. Mean oscillation spaces. We begin by proving that the Besov spaces can be characterized as the mean oscillation spaces in the Bergman metric. We then conclude with some remarks and open questions. Recall that in (2) we have defined the mean oscillation of order $j$ of $f \in \mathscr{H}(B)$.

Proof of 1.12. Recall that for $w \in E_{\zeta},\left|E_{\zeta}\right|^{-1} \approx\left|\operatorname{det} \varphi_{\zeta}^{\prime}(w)\right|^{2}$. Let $\rho<\rho_{0}$ as in the proof of 1.2 (then $\rho$ depends on the size of the lattice in the atomic decomposition). Then for $f \in \mathscr{H}(B)$ we have

$$
\begin{aligned}
\mathrm{MO}_{\rho}^{j} f(\zeta) & \leq \frac{2}{\left|E_{\zeta}\right|} \int_{E_{\zeta}}\left|\Delta_{j} f(w, \zeta)\right| d m(w) \\
& \leq c \int_{E_{\zeta}}\left|\Delta_{j} f(w, \zeta)\right|\left|\operatorname{det} \varphi_{\zeta}^{\prime}(w)\right|^{2} d m(w) \\
& \leq c \int_{E_{0}}\left|\Delta_{j} f\left(\varphi_{\zeta}(w), \zeta\right)\right| d m(w) \\
& \leq c \int_{B}\left|\Delta_{j} f\left(\varphi_{\zeta}(w), \zeta\right)\right| d m(w)
\end{aligned}
$$

Thus, (i) $\Rightarrow$ (ii) follows from 3.1. Conversely, let $C_{\zeta}$ be a function that depends only on $\zeta$. By 3.2 and Cauchy estimates we have that

$$
\begin{aligned}
& \left(1-|\zeta|^{2}\right)^{j}\left|R^{j} f(\zeta)\right| \leq c \int_{E_{0}}\left|\Delta_{j} f\left(\varphi_{\zeta}(w), \zeta\right)-C_{\zeta}\right| d m(w) \\
& \quad=c \int_{E_{\zeta}}\left|\Delta_{j} f(w, \zeta)-C_{\zeta}\right|\left|\operatorname{det} \varphi_{\zeta}^{\prime}(w)\right|^{2} d m(w) \\
& \quad \leq \frac{c}{\left|E_{\zeta}\right|} \int_{E_{\zeta}}\left|\Delta_{j} f(w, \zeta)-C_{\zeta}\right| d m(w)
\end{aligned}
$$

We choose $C_{\zeta}$ to be equal to $\left(\Delta_{j} f(\cdot, \zeta)\right)_{E_{\zeta}}$, and we integrate both sides of (14) with respect to $d \lambda$. This concludes the proof.

Final remarks. Some comments and open questions are in order.

First of all, we expect the result in 1.9 to hold for all $p$ 's, that is, also for $0<p<2$. In this case more delicate techniques seem to be needed.

The results about the generalized Hankel operators could be extended to the weighted Bergman spaces. The same techniques as in the present paper should enable one to prove the corresponding theorems. 
The characterization of Besov spaces as mean oscillation spaces in the Bergman metric probably holds also for $p$ in the range $0<p<1$. Moreover a version of Theorem 1.12 should hold possible for the weighted Besov spaces.

\section{REFERENCES}

[1] S. Axler, The Bergman space, the Bloch space, and commutators of multiplication operators, Duke Math. J., 53 (1986), 315-332.

[2] J. Arazy, S. D. Fisher, S. Janson and J. Peetre, Membership of Hankel operators in unitary ideals, London J. Math., to appear.

[3] J. Arazy, S. D. Fisher and J. Peetre, Hankel operators on weighted Bergman spaces, Amer. J. Math., 110 (1988), 989-1054.

[4] _ Möbius invariant function spaces, J. Reine Angew. Math., 363 (1985), 110-145.

[5] H. P. Boas, Sobolev space projections in strictly pseudoconvex domains, Trans. Amer. Math. Soc., 288 (1985), 227-240.

[6] R. Coifman and R. Rochberg, Representation theorems for harmonic and holomorphic functions in $L^{p}$, Asterisque, 77 (1980), 11-66.

[7] M. Feldman and R. Rochberg, Singular value estimates for commutators and Hankel operators on the unit ball and the Heisenberg group, preprint 1989.

[8] G. H. Hardy, J. E. Littlewood and G. Polya, Inequalities, 2nd ed., Cambridge Univ. Press, London and New York, 1952.

[9] S. Janson and R. Rochberg, Intermediate Hankel operators on the Bergman space, J. Operator Theory, to appear.

[10] S. Janson and T. Wolff, Schatten classes and commutators of singular integral operators, Ark. Mat., 20 (1982), 301-310.

[11] J. Peetre, New Thoughts on Besov Spaces, Duke Univ. Math. Series 1, Durham, NC, 1976.

[12] M. M. Peloso, Möbius invariant spaces on the unit ball, Michigan Math. J., to appear.

[13] L. Peng, R. Rochberg and Z. Wu, Orthogonal polynomials and middle Hankel operators on Bergman spaces, preprint 1990.

[14] W. Rudin, Function Theory on the Unit Ball, Springer-Verlag, New York, 1980.

[15] B. Russo, On the Hausdorff-Young theorem for integral operators, Pacific J. Math., 68 (1977), 241-253.

[16] K. Stroethoff, Besov-type characterisations of the Bloch space, Bull. Austral. Math. Soc., 39 (1989), 405-420.

[17] R. M. Timoney, Bloch functions of several variables, J. Bull. London Math. Soc., 12 (1980), 241-267.

[18] _ B Bloch functions of several variables II, J. Reine Angew. Math., 319 (1980), 1-27.

[19] R. Wallstèn, Hankel operators between weighted Bergman spaces on the unit ball, Ark. Mat., 28 (1990), 183-192.

[20] K. H. Zhu, The Bergman space, the Bloch space, and Gleason's problem, Trans. Amer. Math. Soc., 309 (1988), 260-278.

[21] __ Schatten class Hankel operators on the Bergman spaces of the unit ball, Amer. J. Math., to appear. 
[22] __, Analytic Besov spaces, J. Math. Anal. Appl., to appear.

[23] - Hilbert-Schmidt Hankel operators on the Bergman space, Proc. Amer. Math. Soc., to appear.

Received June 27, 1990.

POLITECNICO DI TORINO

Corso DuCA Degli Abruzzi, 24

10129 TORINO, ITALY 


\title{
PACIFIC JOURNAL OF MATHEMATICS
}

Founded by

\author{
E. F. BECKENBACH (1906-1982) F. WolF (1904-1989)
}

\section{EDITORS}

Sun-Yung A. Chang

(Managing Editor)

University of California

Los Angeles, CA 90024-1555

chang@math.ucla.edu

\section{F. Michael Christ}

University of California

Los Angeles, CA 90024-1555

christ@math.ucla.edu

HeRbert Clemens

University of Utah

Salt Lake City, UT 84112

clemens@math.utah.edu
Thomas ENRIGHT

University of California, San Diego

La Jolla, CA 92093

tenright@ucsd.edu

Nicholas ERCOLANI

University of Arizona

Tucson, AZ 85721

ercolani@math.arizona.edu

\section{R. FINN}

Stanford University

Stanford, CA 94305

finn@gauss.stanford.edu

VAughan F. R. Jones

University of California

Berkeley, CA 94720

vfr@math.berkeley.edu
SteVen KerckhofF

Stanford University

Stanford, CA 94305

spk@gauss.stanford.edu

Martin ScharlemanN University of California Santa Barbara, CA 93106 mgscharl@henri.ucsb.edu

\section{Harold Stark}

University of California, San Diego La Jolla, CA 92093

V. S. VARADARAJAN University of California Los Angeles, CA 90024-1555 vsv@math.ucla.edu

\section{SUPPORTING INSTITUTIONS}

UNIVERSITY OF ARIZONA

UNIVERSITY OF BRITISH COLUMBIA

CALIFORNIA INSTITUTE OF TECHNOLOGY

UNIVERSITY OF CALIFORNIA

UNIVERSITY OF MONTANA

UNIVERSITY OF NEVADA, RENO

NEW MEXICO STATE UNIVERSITY

OREGON STATE UNIVERSITY
UNIVERSITY OF OREGON

UNIVERSITY OF SOUTHERN CALIFORNIA

STANFORD UNIVERSITY

UNIVERSITY OF HAWAII

UNIVERSITY OF UTAH

WASHINGTON STATE UNIVERSITY

UNIVERSITY OF WASHINGTON 


\section{PACIFIC JOURNAL OF MATHEMATICS}

Volume $161 \quad$ No. $1 \quad$ November 1993

Tangential and normal Euler numbers, complex points, and

singularities of projections for oriented surfaces in four-space

ThOMAS FRANCIS BANCHOFF and FrANK ALBERT FARRIS

Studying links via closed braids. III. Classifying links which are closed 3-braids

JOAN Birman and William W. MEnAsCo

Dehn functions of groups and extensions of complexes

STEPHEN GARY BRICK

Contact structures on $(n-1)$-connected $(2 n+1)$-manifolds

\section{HANSJÖRG GEIGES}

The relative Nielsen number and boundary-preserving surface maps

MiCHAEL R. KELLY

Besov spaces, mean oscillation, and generalized Hankel operators

\section{MARCo Maria PELOSO}

On the nonoccurrence of the Coxeter graphs $\beta_{2 n+1}, D_{2 n+1}$ and $E_{7}$ as 185 the principal graph of an inclusion of $\mathrm{II}_{1}$ factors

ViakALATHUR SHANKAR SUNDER and A. K. ViJAYARAJAN 